Non-convergence of formal integrals of motion

This article has been downloaded from IOPscience. Please scroll down to see the full text article.

2003 J. Phys. A: Math. Gen. 368639

(http://iopscience.iop.org/0305-4470/36/32/306)

View the table of contents for this issue, or go to the journal homepage for more

Download details:

IP Address: 159.149.103.9

The article was downloaded on 04/06/2013 at 17:09

Please note that terms and conditions apply. 


\title{
Non-convergence of formal integrals of motion
}

\author{
G Contopoulos ${ }^{1}$, C Efthymiopoulos ${ }^{1,2}$ and A Giorgilli ${ }^{3}$ \\ ${ }^{1}$ Research Center for Astronomy, Academy of Athens, Anagnostopoulou 14, 10673 Athens, \\ Greece \\ 2 Department of Statistics, University of the Aegean, Karlovossi, Samos, Greece \\ ${ }^{3}$ Department of Applied Mathematics, University of Milano Bicocca, via Bicocca degli \\ Arcimboldi 8, 20126 Milano, Italy
}

Received 30 January 2003, in final form 2 June 2003

Published 29 July 2003

Online at stacks.iop.org/JPhysA/36/8639

\begin{abstract}
We consider formal integrals of motion in 2D Hamiltonian dynamical systems, calculated with the normal form method of Giorgilli (1979 Comput. Phys. Commun. 16 331). Three different non-integrable and one integrable systems are considered. The time variation $D I$ of the formal integral $I$ is found as a function of the order of truncation $N$ of the integral series. An optimal order of truncation is found from the minima of the variations $D I$. The level lines of the integral I, representing theoretical invariant curves on a Poincaré surface of section, are compared with the real invariant curves. When chaos is limited, excellent agreement is found between the theoretical and the real invariant curves, if the order of truncation is close to the optimal order. The agreement is poor (a) far from the optimal order and (b) when chaos is pronounced. The optimal order, calculated as a function of the distance $R$ from the origin, decreases when $R$ increases. The decrease is rather smooth in the 1:1 resonance, but it has abrupt steps in the case of a higher order (4:3) resonance. In the case of an integrable Hamiltonian, a formal integral $I_{\mathrm{F}}$ is found which is a function of the exact integral $I$ and of the Hamiltonian, given as power series of the canonical variables. The series converges only within a domain of convergence. The radius of convergence along a particular direction is calculated with the d'Alembert and Cauchy methods. The theoretical invariant curves agree with the real invariant curves only within the domain of convergence of $I_{\mathrm{F}}$. In the case of non-integrable Hamiltonians, we calculate 'pseudo-radii of convergence' that tend to zero as the order of truncation $N$ increases.
\end{abstract}

PACS numbers: $05.45 .-\mathrm{a}, 45.10 .-\mathrm{b}$

\section{Introduction}

Formal expansions of first integrals for Hamiltonian systems in the neighbourhood of an elliptic equilibrium were first introduced by Whittaker (1916, 1937), Cherry (1924a, b) and Birkhoff (1927). However, the question concerning the convergence of the formal expansion was left 
unsettled by these authors. A result stating that divergence generically occurs was proved by Siegel (1941). On the other hand, Littlewood (1959a, b) exploited the asymptotic character of the formal expansions in order to prove a stability result over very long times for the Lagrangian points in the restricted problem of three bodies. Contopoulos (1960) introduced the formal expansion of first integrals in the study of galactic models, and wrote the first implementation of a computer program that could perform the formal expansions (Contopoulos and Moutsoulas 1965). Similar programs were later implemented, using different algorithms, by Gustavson (1966) and by Giorgilli (1979). Many applications of these quantities have been given over the years. Furthermore, a recent renewal of interest is due to the applications to self-consistent galactic models generated by $N$-body simulations (Contopoulos et al 2000) and to the search for realistic stability results for the Trojan asteroids (Giorgilli et al 1989, Celletti and Giorgilli 1991, Giorgilli and Skokos 1997, Skokos and Dokoumetzidis 2001).

The interest for applications has also renewed the interest for the problem of investigating the limit of validity of the formal expansions of first integrals. For, it has been remarked by Gustavson (1966) that the functions obtained by truncating the formal series at some low order may reproduce quite well the orbits of the system in some ordered regions of the phase space. On the other hand, Roels and Hénon (1967) with a careful investigation produced evidence of the asymptotic character of the formal expansions for an area preserving mapping. More recently, Kaluza and Robnik (1992) calculated the second integral of motion of the Hénon and Heiles system (1964) up to terms of degree 14, and found an apparently good convergence.

Now, the main purpose of this paper is precisely to investigate the usefulness of the formal series expansions of first integrals as due to the apparent convergence caused by the asymptotic behaviour of the series. Precisely, by truncating the series expansion of the first integral to different orders, we produce numerical evidence of the existence of an optimal order where the variation of the truncated integral along particular orbits of the system is minimal. For lower and higher order truncations, the variations of the truncated integrals are larger, and the series turn out to be typically divergent if one lets the truncation order go to infinity; this is in agreement with the theorem of Siegel.

The existence of an optimal truncation order may be understood in the framework of Nekhoroshev's theory (Nekhoroshev 1977). An analytical estimate of the best order of truncation in the case of an elliptic equilibrium may be found in Giorgilli (1988). Let us assume that the harmonic frequencies $\left(\omega_{1}, \ldots, \omega_{n}\right)$ of the linearized unperturbed system satisfy a diophantine condition

$$
\left|m_{1} \omega_{1}+\cdots+m_{n} \omega_{n}\right|>\frac{K}{|m|^{\tau}}
$$

for some constants $\tau \geqslant n-1$ and $K>0$ and for all non-zero integer vectors $m=$ $\left(m_{1}, \ldots, m_{n}\right)$, where $|m|=\left|m_{1}\right|+\cdots+\left|m_{n}\right|$. Consider a ball of radius $R$ centred at the origin of $\mathbf{R}^{2 n}$. Then an estimate of the optimal order $N$ of truncation of the formal series is given by

$$
\log N \simeq-\frac{1}{\tau} \log \left(\frac{R}{R_{*}}\right)
$$

where $R_{*}$ is a constant depending on $K$, the number $n$ of degrees of freedom and the size of the non-linear coupling term. We emphasize that according to the latter formula the optimal order $N$ is expected to decrease when the radius $R$ of the domain is increased.

Now, the estimate of equation (2) relies on some upper bound estimates on the coefficients of the series, and cannot capture all details of the dependence of $N$ on $R$. The stronger reason is that the diophantine estimate (1) is just a uniform lower bound on the smallness of $\left|m_{1} \omega_{1}+\cdots+m_{n} \omega_{n}\right|$ — the so-called small divisors — which applies to most real vectors $\omega$ 
(in measure sense), but veils the fact that very small divisors are quite rare. Moreover, in the case of resonant frequencies, i.e., frequencies $\omega$ satisfying $m_{1} \omega_{1}+\cdots+m_{n} \omega_{n}=0$ for some integer vector $m$, the diophantine condition must be replaced by a weaker one, and the construction of formal integrals must be performed by taking into account the resonance: see for instance Contopoulos (1963), Gustavson (1966) or Giorgilli and Galgani (1978). In particular, if one considers a resonant case of two degrees of freedom, then the two frequencies are multiples of a given non-zero quantity, so that the expression $\left|m_{1} \omega_{1}+m_{2} \omega_{2}\right|$ is either zero or bounded from below by a constant. Thus, no small divisors appear in the perturbation series for the first integral. Nevertheless, the theorem of Siegel still applies, and the divergence seems to be due to the fast increase of the coefficients generated by the formal algorithm of construction of the series. The theoretical estimates for this case are still expressed by (2), with $\tau=1$. We conclude that the details of the dependence of the optimal order of truncation $N$ on the size $R$ of the domain can be only determined via numerical investigations.

In this paper we perform a series of experiments for 2D non-linear Hamiltonian systems in the neighbourhood of an elliptic equilibrium, and for two different resonances, namely: (a) $\omega_{1}: \omega_{2}=1: 1$ and (b) $\omega_{1}: \omega_{2}=4: 3$. All systems considered are of the form

$$
H \equiv \frac{1}{2}\left(\dot{x}^{2}+\dot{y}^{2}+A x^{2}+B y^{2}\right)+H_{1}
$$

where $H_{1}$ is a polynomial of degree at least 3 .

In section 2 we consider the Hamiltonian

$$
H \equiv \frac{1}{2}\left(\dot{x}^{2}+\dot{y}^{2}+x^{2}+y^{2}\right)+x^{2} y^{2}=E
$$

that has $A=B=1$ and a quartic non-linear coupling.

In section 3 we consider the case investigated by Hénon and Heiles, namely $A=B=1$ and

$$
H \equiv \frac{1}{2}\left(\dot{x}^{2}+\dot{y}^{2}+x^{2}+y^{2}\right)+x^{2} y-\frac{1}{3} y^{3}=E .
$$

In section 4 we consider the Hamiltonian

$$
H \equiv \frac{1}{2}\left(\dot{x}^{2}+\dot{y}^{2}+A x^{2}+B y^{2}\right)-x y^{2}=E
$$

with $A=B=1$ (case (6a)) and $A=1.6, B=0.9$ (case (6b)). In the latter case the frequencies are resonant, with a ratio $4: 3$.

In section 5 we consider the integrable case $A=B=1$ and

$$
H \equiv \frac{1}{2}\left(\dot{x}^{2}+\dot{y}^{2}+x^{2}+y^{2}\right)-x^{2} y-2 y^{3}=E
$$

found by Bountis et al (1982). In this case an explicit polynomial expression of a first integral independent of the Hamiltonian is known.

In all cases a formal first integral independent of the Hamiltonian is calculated using the computer program implemented by Giorgilli (1979), making the appropriate expansion for the resonance $\sqrt{A}: \sqrt{B}=1: 1$ in cases corresponding to equations (4), (5), (6a) and (7), and for the resonance $\sqrt{A}: \sqrt{B}=4: 3$ in case (6b). A first quantity that can be defined numerically is an overall optimal order evaluated by comparing orbits of the Poincaré mapping on a surface of section and the level surfaces of the first integral on the same surface (Contopoulos and Moutsoulas 1965, Gustavson 1966). A particular calculation refers to the periodic orbits found by means of the truncated formal integrals as compared with the exact periodic orbits (Contopoulos 1968).

Such calculations may give the feeling that the formal integrals are in fact convergent, because the formal results give good approximations to real invariant curves, or they seem to converge to some final form even when the true orbits exhibit a chaotic behaviour. This was indeed the remark of Kaluza and Robnik concerning the Hamiltonian (5) of Hénon and Heiles. 
However, we will show that if one pushes the expansions to higher orders, then the divergence of the formal series shows up, since the results become worse and worse.

A more quantitative evaluation of the optimal order $N$ is performed by determining $N$ as the order at which the variations $D I$ of the formal integral $I$ along an orbit of the system are minimal. In all non-integrable cases with a 1:1 resonance (cases (4), (5) and (6a)) $N$ turns out to be a rather smoothly decreasing function of $R$. However, in the case $(6 \mathrm{~b})$ of the resonance 4:3 we find that $N(R)$ decreases with abrupt steps. These results have been found also in other numerical experiments, not described in detail here. Thus, the present exploration seems to reflect the generic behaviour of $N(R)$.

As regards the integrable system (7), Kaluza and Robnik (1992) noted that a formal integral calculated via the Birkhoff normal form did not reproduce exactly the real invariant curves of the Poincaré surface of section. This fact is explained in section 5. It is found that the formal integral defined by the normal form method is a function of the Hamiltonian and of the known polynomial first integral, but is expressed as a power series in the canonical variables that need not converge in the whole phase space. A domain of convergence of this function is estimated numerically by considering the radii of convergence along particular directions, as evaluated with the classical methods of analysis (section 6). It is then shown that the theoretical invariant curves derived by means of the formal integral coincide with the true invariant curves only within the domain of convergence of the formal series for the first integral. We emphasize that approximate radii of convergence may be estimated for the non-integrable cases as well, but they appear to tend slowly to zero as the truncation order increases.

The general conclusions from these experiments are summarized in section 7 .

\section{Hamiltonian (4)}

The structure of orbits in this Hamiltonian has been studied in detail by Contopoulos et al (1994). This Hamiltonian does not have an escape energy, therefore the orbits remain bounded for arbitrarily large energies. The $y$-axis is a periodic orbit that is represented by the central point $(x=\dot{x}=0)$ on the surface of section $(x, \dot{x})$. This orbit is stable for small energies. As the energy increases, this orbit becomes unstable and then alternatively stable and unstable an infinite number of times.

In the present paper we consider a formal second integral, for small values of the energy $H=E$.

The Poincare map of the Hamiltonian (4) on the surface of section (SOS) $y=0$ for the energies $E=0.4$ and 0.8 is shown in figures $1(a)$ and 2(a) respectively. Besides the central periodic orbit $(x=\dot{x}=0)$, there are three more basic periodic orbits:

(1) The orbits $B_{1}, B_{2}$ at $x=0, \dot{x}= \pm \dot{x}_{B}$ above and below the centre. These orbits are stable for small energies and unstable for larger energies $(E>2.367)$.

(2) The orbits $C_{1}, C_{2}$ at $\dot{x}=0, x= \pm x_{C}$ on the left and right of the centre. These orbits are always unstable and generate more and more chaos around them as the energy increases.

(3) The orbit $y=\dot{y}=0$, which is represented in figures $1(a)$ and 2(a) by the boundary $\dot{x}^{2}+x^{2}=2 E$. This has the same properties as the central orbit $(x=\dot{x}=0)$, and changes from stability to instability at the same values of $E$.

The second formal integral in this case is

$\Phi=\frac{1}{2}\left(\dot{x}^{2}+\dot{y}^{2}+x^{2}+y^{2}\right)+\frac{5}{8} x^{2} y^{2}-\frac{1}{8} x^{2} \dot{y}^{2}-\frac{1}{2} x y \dot{x} \dot{y}-\frac{1}{8} y^{2} \dot{x}^{2}-\frac{3}{8} \dot{x}^{2} \dot{y}^{2}+\cdots$.

It contains only even order terms because of the symmetry of the potential. 

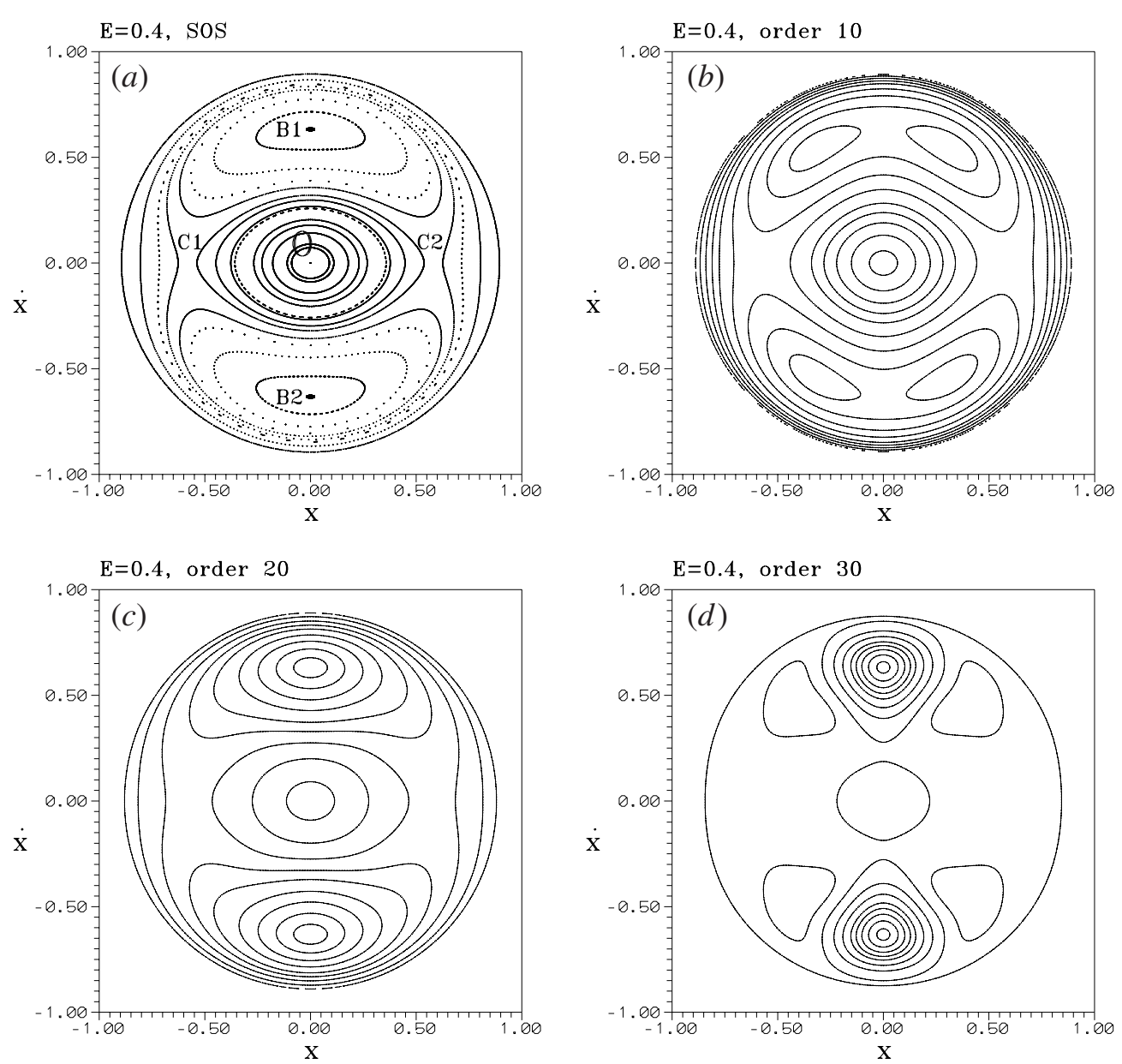

Figure 1. Poincaré SOS $(x, \dot{x})$ (i.e. $y=0$ ) for the Hamiltonian (4) and $E=0.4$.

The theoretical invariant curves are derived by introducing the value

$$
\dot{y}^{2}=2 E-\dot{x}^{2}-x^{2}
$$

derived from equation (4) for $y=0$, into equation (8).

Then, if $y=0$ we derive

$$
2 \Phi=2 E+\frac{1}{8}\left(x^{2}+3 \dot{x}^{2}\right)\left(2 E-\dot{x}^{2}-x^{2}\right)+\cdots .
$$

For various initial conditions $(x, \dot{x})$ we derive curves like those of figures $1(b)$ and $(c)$ for $E=0.4$ and various truncations of the second integral. Namely the invariant curves for a truncation at order $N=10$ (figure $1(b)$ ) have some similarities with the real invariant curves of figure 1(a), but also some important differences (e.g. the orbits $B_{1}, B_{2}$ are unstable in figure $1(b)$ ). The agreement is much better when the truncation is at order $N=20$ (figure $1(c)$ ) but the deviations are very large if $N=30$ (figure $1(d)$ ).

In the case $E=0.8$ the Poincaré SOS is shown in figure 2(a). In this case we see chaotic regions around the unstable periodic orbits $C_{1}$ and $C_{2}$ which also surround the central invariant curves and the invariant curves around $B_{1}$ and $B_{2}$. The theoretical invariant curves when the second integral is truncated at order $N=8$ (figure $2(b)$ ) are qualitatively similar, but without any chaos. The results are completely different when the truncation is at order $N=10$ 

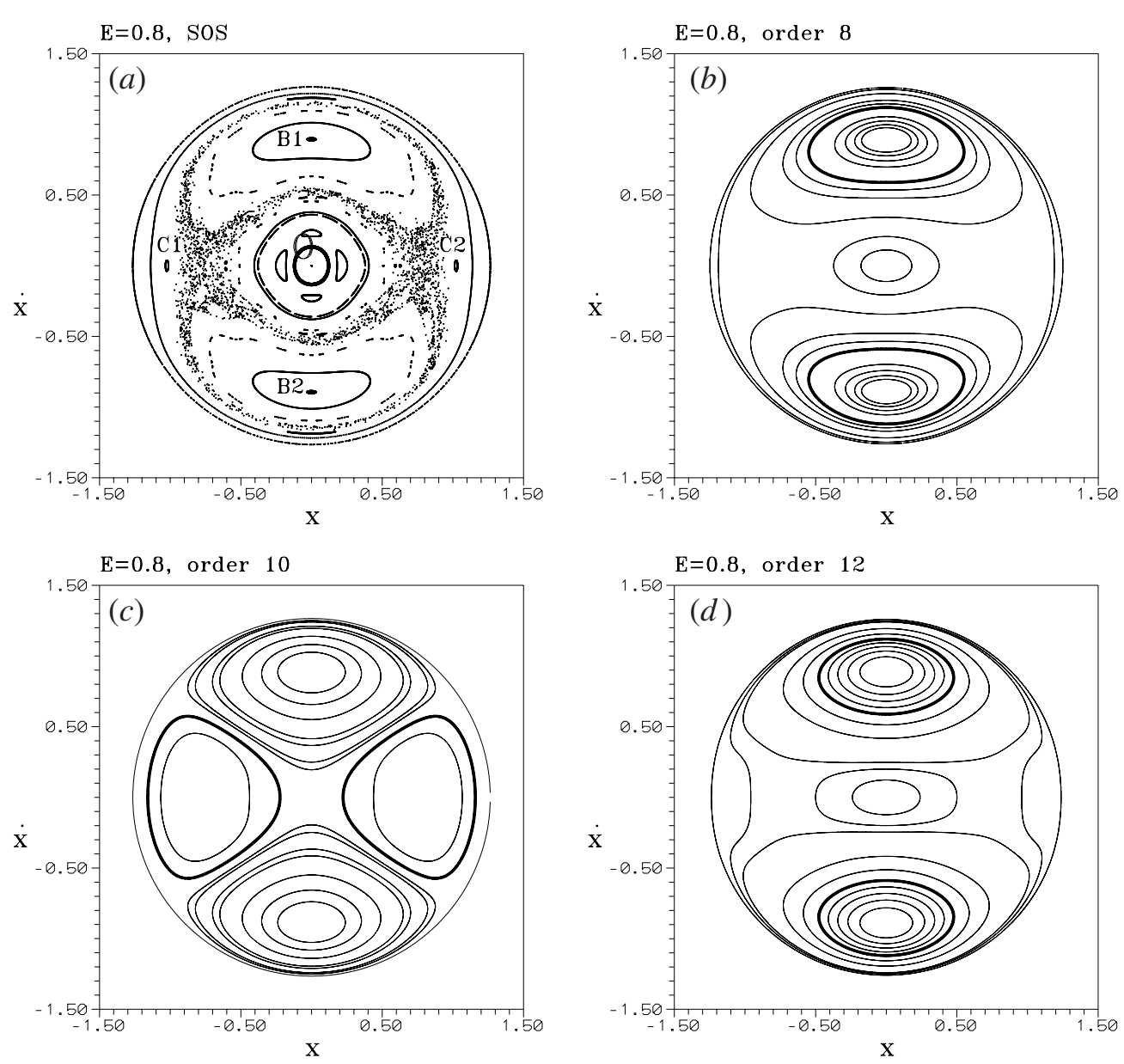

Figure 2. As in figure 1 for $E=0.8$. (a) Numerical results (chaos appears around $C_{1}$ and $C_{2}$ ). Theoretical invariant curves for: $(b) N=8,(c) N=10$ and $(d) N=12$.

(figure 2(c)) in which case the theoretical periodic orbit at $\mathrm{C} 1$ and $\mathrm{C} 2$ is stable while the theoretical central periodic orbit at $\mathrm{O}$ is unstable. Agreement is found again at order $N=12$ (figure $2(d)$ ). There is also some agreement at orders $N=16, N=20$ while the agreement is bad at orders $N=14, N=18$. Bad results also appear for larger orders of truncation.

An estimate of the constancy of the second integral is made by giving the variation of the integral $D I=I_{\max }-I_{\min }$ truncated at various orders, along certain orbits. In figure 3 we give the variation $D I$ as a function of the order of truncation $N$ for orbits with various initial conditions along the $x$-axis with $y=\dot{x}=0$ and $\dot{y}$ specified by the constant energy condition $E=0.4$. We see that $D I$ decreases smoothly with increasing $N$ up to an optimal value of $N$; then, for larger $N$, the error $D I$ increases.

When $x$ increases up to a certain maximum, the optimal value of $N$ decreases and the minimum value of $D I$ increases. For larger $x$ the optimal $N$ increases again and the minimum $D I$ decreases. This is due to the fact that these values of $x$ are close to the boundary $\dot{x}^{2}+x^{2}=2 E$, which represents the stable periodic orbit $y=0$, which has the same properties as the central periodic orbit $x=0$. The uppermost curve refers to $x$ close to the unstable invariant point $C_{2}$. 


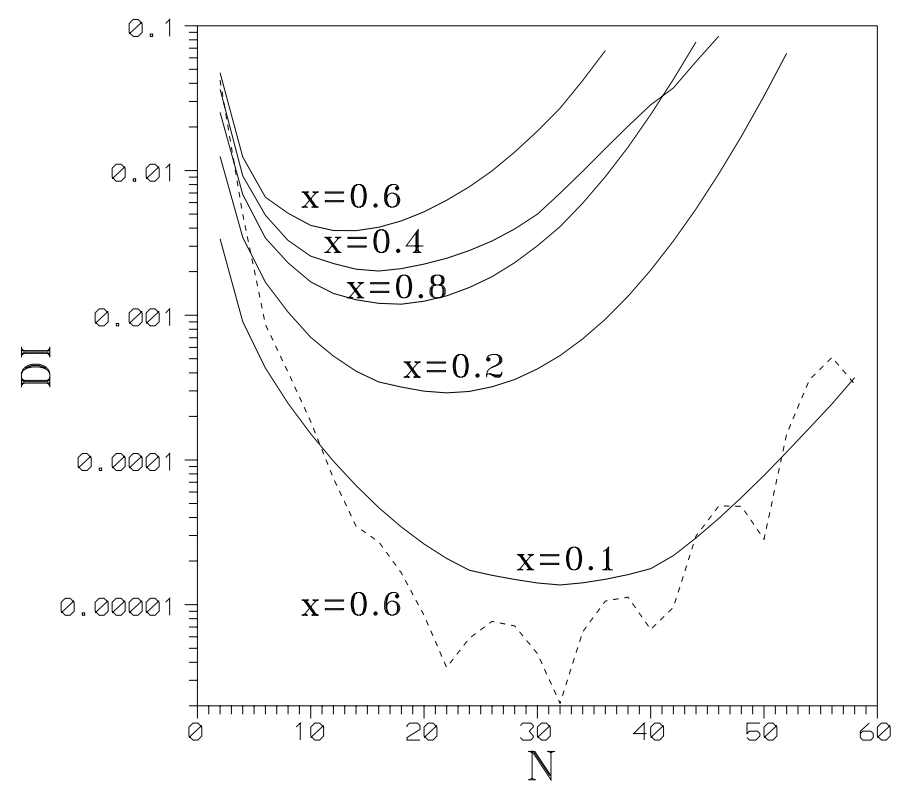

Figure 3. The variation $D I=I_{\max }-I_{\min }$ of the formal integral $I$, in the case of the Hamiltonian (4) truncated at various orders $N$, for various values of $x$, and $y=\dot{x}=0$ ( $E=0.4=$ const). The dashed line corresponds to the curve for $x=0.6$ but for a much shorter integration time.

For $x$ close to the points $C_{1}$ and $C_{2}$ (e.g. $x=0.6$ ), a reliable estimate of $D I$ requires a long time of calculation ( $t=2 \times 10^{4}$, solid line $x=0.6$ in figure 3 ). If the time is short $\left(t=10^{3}\right.$, dashed line with $x=0.6$ ), then the values of $D I$ vary in an oscillatory way and the minimum value of $D I$ is smaller. On the other hand, for smaller $x$ (e.g. $x=0.2$ in figure 3) and also for larger $x$ the increase of the time of calculation does not appreciably change the results. Therefore, one has to find the correct minimum $D I$ by checking if its variations remain the same as the time of integration of an orbit increases.

Figure 4 gives the minimum variation $D I$ as a function of $\dot{x}$ for various values of the energy $E$. We find that, as the distance from the centre increases up to a maximum, the variation $D I$ becomes larger. For $E=0.4,0.6$ and 0.8 and for smaller $\dot{x}$, the variations of $\log (D I)$ are almost linear with respect to $\log (\dot{x})$ but for larger $\dot{x}$ they grow less than linearly. For larger $\dot{x}$ the variations decrease again. This is explained because the invariant curves for $\dot{x}>0.4$ are closed around $B_{1}$, thus a value of $\dot{x}>\dot{x}_{B}$ gives the same orbit with a corresponding value of $\dot{x}<\dot{x}_{B}$. The maximum $D I$ as a function of $\dot{x}$ has a maximum at $\dot{x}=\dot{x}_{B}$, corresponding to the stable periodic orbit $B_{1}$. However, for $E=0.2, D I$ has a local minimum at $\dot{x}_{B}$. The transition from a maximum to a local minimum occurs near $E=0.27$.

Figure 5 gives the optimal order $N$, as a function of the distance $R$ from the centre along the line $x=y$ and $\dot{x}=\dot{y}=0$. Our calculations of the integral series were stopped for $R<0.58$ at order 60, which is the highest optimal order found in this figure. For $R$ larger than $R=0.58$, we find that the optimal order $N$ decreases as $R$ increases, approximately as a power law. This fact is in agreement with the asymptotic character of the series, predicted by Nekhoroshev's (1977) theory. (The plateau for small values of $R$ is due to the truncation of the series at order 60.) We also calculated the minimum $D I$ as a function of the optimal order $N$. DI decreases with increasing $N$ and the variation of $\log (D I)$ with $\log N$ is almost linear. 


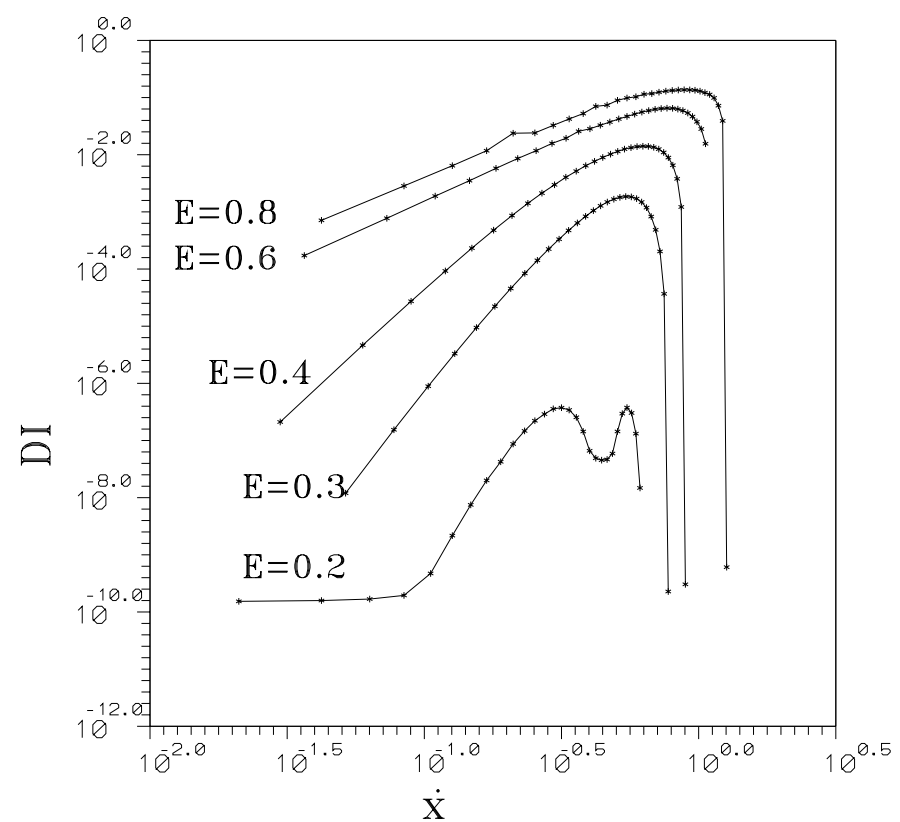

Figure 4. The variation of the minimum $D I$ as a function of $\dot{x}$ for $x=y=0$ and various values of the energy $E$.

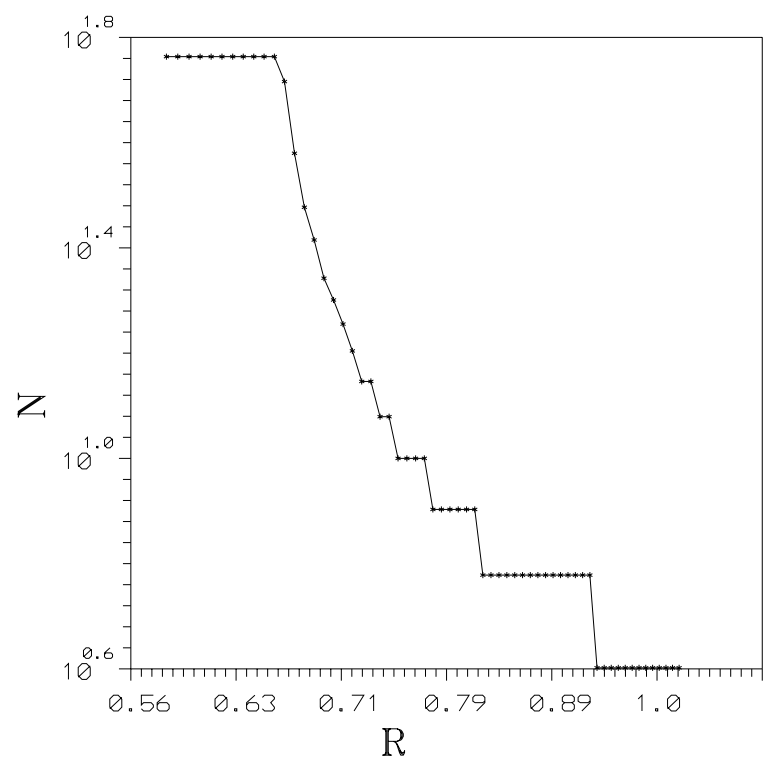

Figure 5. The variation of the optimal order $N$ as a function of $R$.

\section{Hénon-Heiles case (5)}

This model has been used extensively since the original Hénon-Heiles paper (1964). It is well known that for energies smaller than a critical value $E_{\text {crit }}=1 / 12$, the chaotic regions are very 

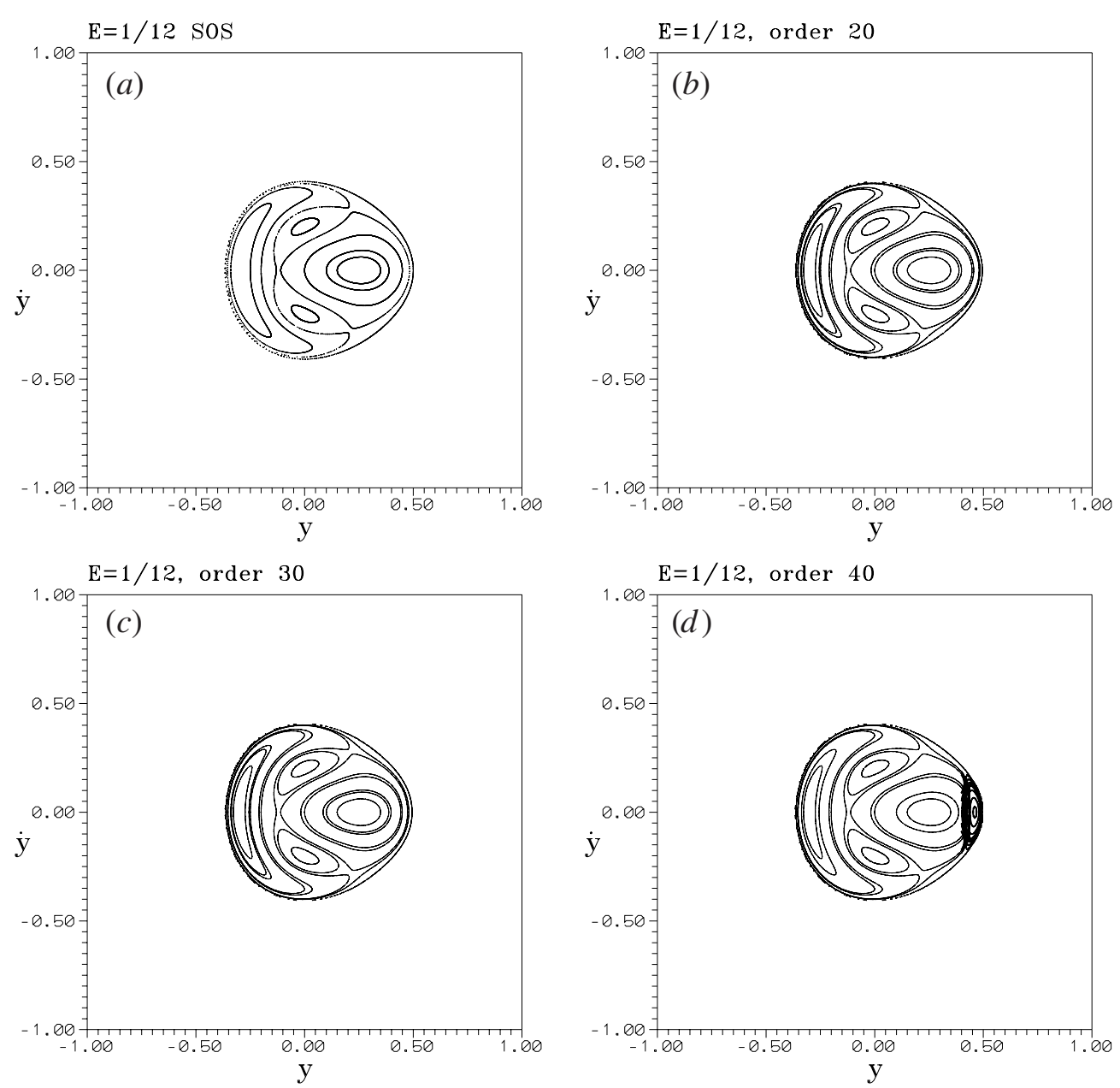

Figure 6. Poincaré surfaces of section (as in figure 1) for the Hénon-Heiles Hamiltonian (5) and $E=1 / 12$. (a) Numerical orbits. Invariant curves derived from the second integral truncated at order: $(b) N=20,(c) N=30$ and $(d) N=40$.

small (figure $6(a)$ ). For a larger value $E=1 / 8$, chaos is appreciable (figure $7(a)$ ) and for still larger energy $(E=1 / 6)$ chaos is dominant.

A comparison between the exact invariant curves on a Poincare SOS and the invariant curves calculated by means of a truncated second integral was made by Kaluza and Robnik (1992). However, these authors stopped their calculations at a maximum order of truncation $N=14$. They found that in the case $E=1 / 12$, the theoretical invariant curves represent quite accurately the real invariant curves. No evidence for divergence was found. In the case $E=1 / 8$ the theoretical invariant curves seemed to converge as the order of truncation approached $N=14$, although in some cases the convergence was slow. On the other hand, the theoretical invariant curves could not represent the chaotic domains in the strongly chaotic case $E=1 / 6$. Nevertheless, convergence seemed to exist even in some chaotic regions. Kaluza and Robnik (1992) noted that for small energies the second integral seemed to converge, while for larger energies the second integral might still converge in some regions. 

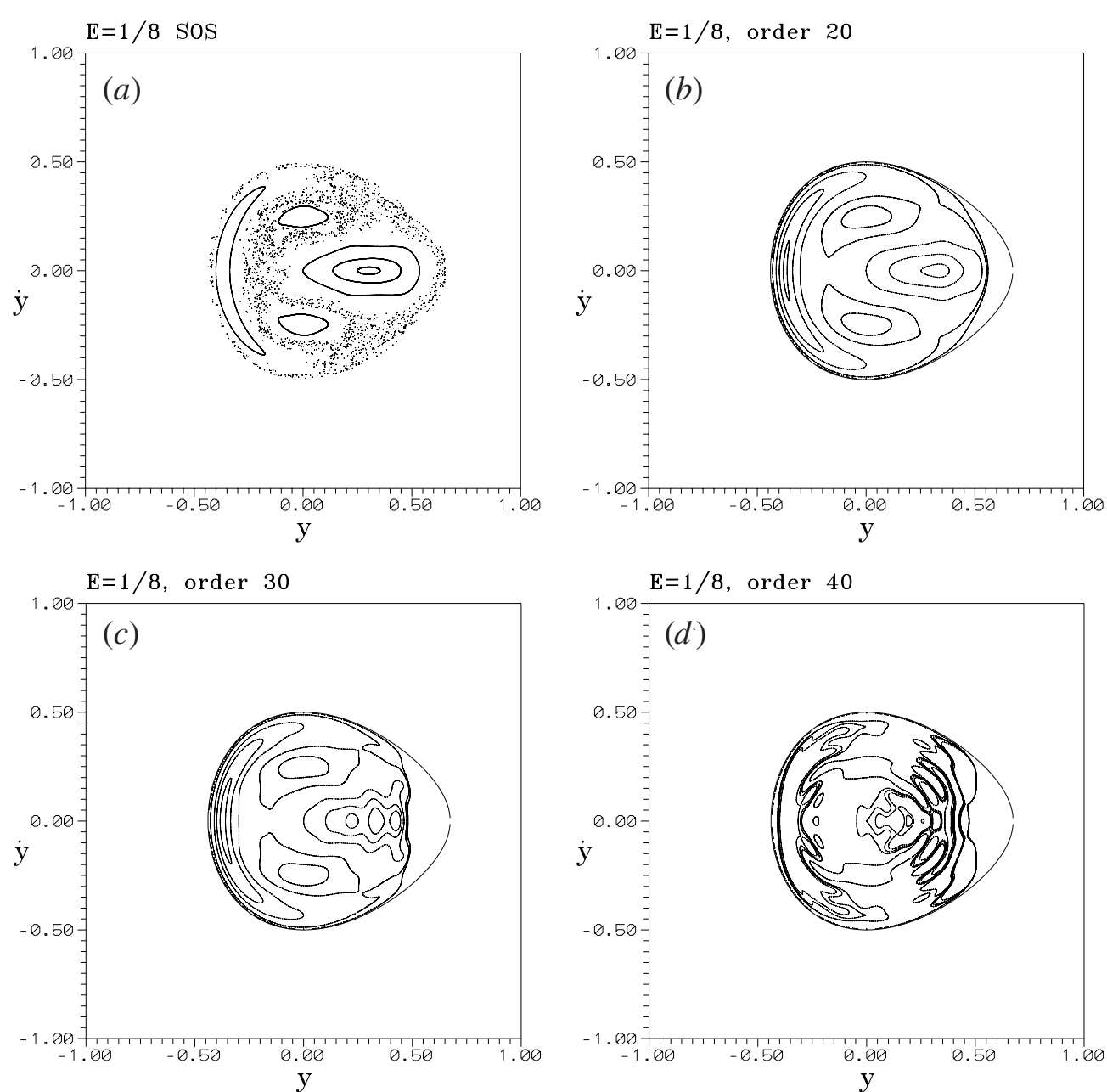

Figure 7. As in figure 6 for $E=1 / 8$. (a) Numerical results (some chaos appears near the unstable periodic orbits). Invariant curves for: $(b) N=20,(c) N=30$ and $(d) N=40$.

However, the non-convergence of the second integral is manifest even in the case $E=1 / 12$ (and also $E=1 / 8$ ) if we truncate the second integral at higher orders. In figures $6(b),(c)$ and $(d)$ we show the theoretical invariant curves when the second integral is truncated at orders $N=20, N=30$ and $N=40$, respectively. We see that the agreement with the real invariant curves of figure $6(a)$ is good for truncation orders $N=20$ and $N=30$ but it is inappropriate for relatively large $x$ if $N=40$.

In the case $E=1 / 8$ the agreement with the SOS (figure 7(a)) is fair (except in the chaotic domain) for a truncation order $N=20$ (figure 7(b)) but it is bad for $N=30$ (figure $7(c)$ ) and still worse for $N=40$ (figure $7(d)$ ).

Finally, in the case $E=1 / 6$ chaos is dominant. In this case we find that all truncations give very bad results, as expected, because most of the phase space is covered by chaotic orbits.

The variations $D I$ as functions of the order $N$ of truncation, for points with increasing distance from the centre $(0,0)$ in the Hamiltonian $(5)$, show a similar qualitative behaviour as 


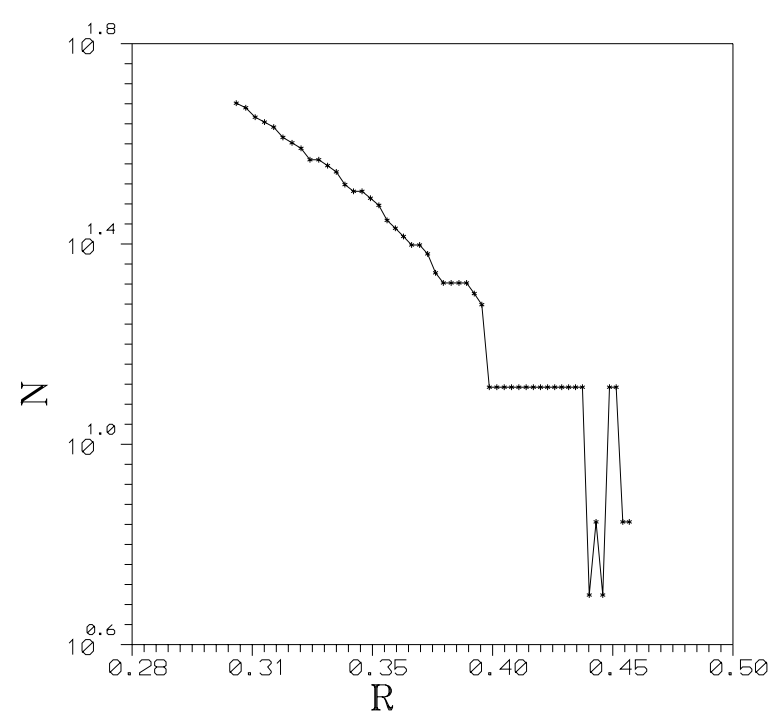

Figure 8. As in figure 5 for the Hamiltonian (5).

in figure 3 for the Hamiltonian (4). Namely, as the order increases the variation $D I$ decreases initially to a minimum variation for an optimal order of truncation $N$. Then, $D I$ increases for $N$ larger than this optimal order.

The optimal order $N$ as a function of the distance from the centre is given in figure 8 . The order $N$ decreases as the distance $R$ increases, in agreement again with the Nekhoroshev theory. In this case, the decrease is rather smooth until $R$ reaches high values corresponding to the initial conditions of chaotic orbits. In fact, we can define an average logarithmic slope in the smooth part of the curve of figure 8 .

The minimum $D I$ for the optimal $N$ decreases with increasing $N$. The decrease of $D I$ with $N$ is smooth, and has a good exponential slope.

\section{Hamiltonian (6)}

The case (6a) $(A=B=1)$ has been studied in detail by Contopoulos and Moutsoulas (1965). For small energies (e.g. $E=0.05$, figure $9(a)$ ) there is practically no chaos. The invariant curves close either around the unstable central invariant point $A$, or around one of the stable invariant points $B$ or $C$.

For larger energy (e.g. $E=0.1$, figure $9(c)$ ) there is some chaos around the unstable orbit $A$, but there are still closed invariant curves around $A, B$ or $C$.

The 'third' integral in this case has a resonant form (Contopoulos and Moutsoulas 1965). The level lines of the 'third' integral, truncated at order 20, are given in figures $9(b)$ and $(d)$. The agreement with the corresponding empirical invariant curves (figures $9(a)$ and $(c)$ ) is very good, except in the chaotic domain near the unstable periodic orbit in the case $E=0.1$ (figures $9(c)$ and $(d)$ ).

The variation $D I$ of the integral $I$ truncated at various orders $N$, as a function of $N$ for various values of $R$ (with $\dot{x}=\dot{y}=0$ ), shows the same qualitative behaviour as that of figure 3 for the Hamiltonian (4). Namely, as $N$ increases the values of $D I$ initially decrease, but for larger $N$ they increase. The minimum $D I$ occurs at particular values of $N$ (figure 10) that decrease rather smoothly as $R$ increases. 
(a)

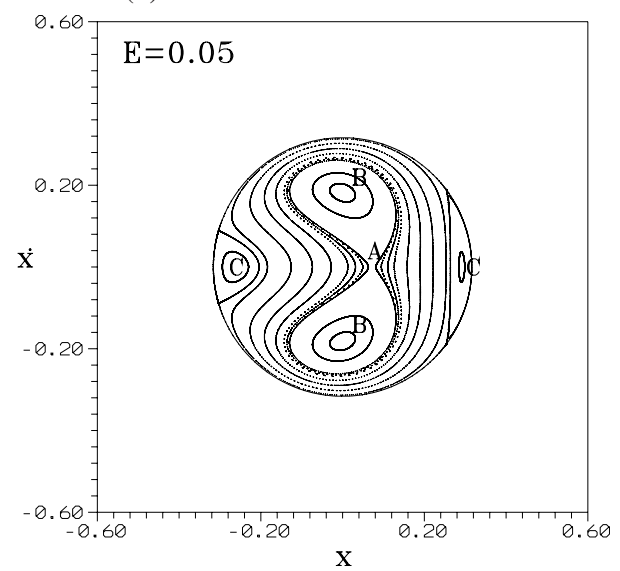

(c)

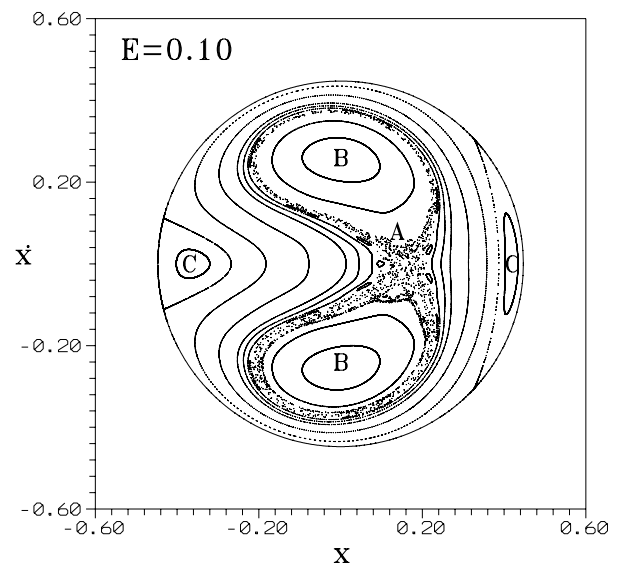

$(b)$

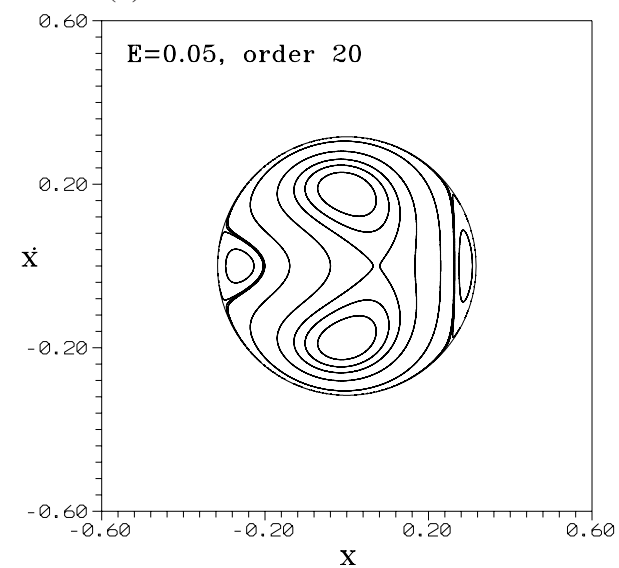

(d)

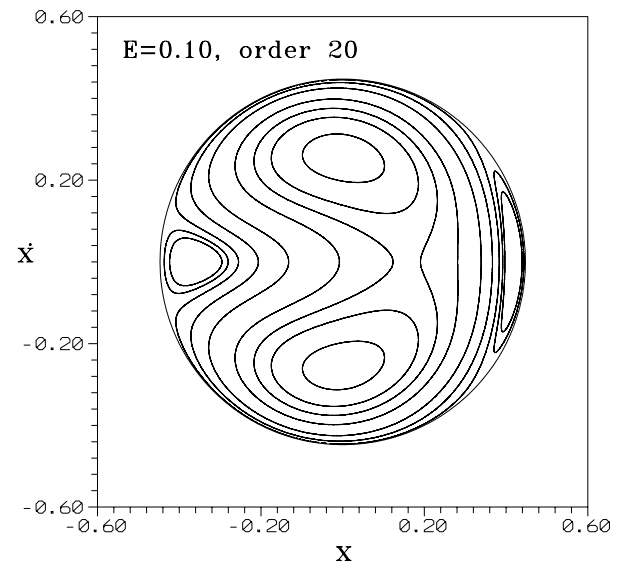

Figure 9. Poincaré surfaces of section (as in figure 1) for the Hamiltonian (6) with $A=B=1$ and $(a) E=0.05,(c) E=0.10$. (b) and $(d)$ are the corresponding level curves of the second integral truncated at order $N=20$.

The Hamiltonian (6) for $A=1.6, B=0.9$ (case (6b)) has been studied extensively by Contopoulos $(1960,1963)$. In this case the Hamiltonian is not symmetric around the point $x=0$. It has a stable central periodic orbit for relatively small values of $E$, as in figures 11(a), $(b)$ and $(c)$. (For larger $E$ this orbit becomes unstable.) Because of the resonance $\sqrt{A} / \sqrt{B}=4 / 3$, there are also three islands of stability around the central periodic orbit. There is also a triple unstable periodic orbit represented by three points between the three resonant islands. This unstable orbit generates chaos in its neighbourhood by its homoclinic tangle. The chaotic regions are very small in figures $11(a)$ and $(b)$, but they are large in figure 11(c).

Figure 12 gives the variation $D I$ of the integral $I$, as a function of $N$, for various values of $x$ (and $\dot{x}=\dot{y}=0$ ). The values of $D I$ initially decrease on average with $N$, while later, for larger $N$, they increase on average. However, there are some local minima of $D I$ at particular values of $N$. In figure 12 we notice three main minima for $N=N_{\text {res }}=14,30$ and 43, independently of the value of $x$. 


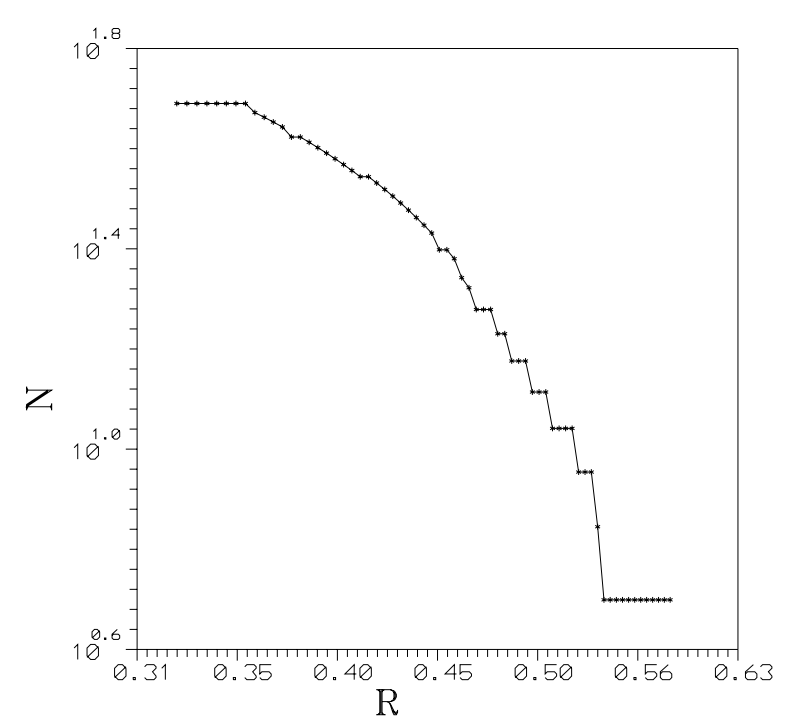

Figure 10. As in figure 5 for the Hamiltonian (6) with $A=B=1$.

Figure 13 gives the optimal truncation order of the integral $I$ as a function of $R=\sqrt{x^{2}+y^{2}}$ for various initial conditions along the line $x=y$ and $\dot{x}=\dot{y}=0$. We find that $N$ is a decreasing function of $R$. In this case the decrease is not smooth but abrupt. Namely, for $0.345<R<0.45$, the optimal order of truncation is 43 . Then for $0.45<R<0.49, N$ goes abruptly to the value $N=30$. For still larger $R$, i.e. $R>0.49, N$ falls abruptly again to the value $N=14$. The minimum $D I$ decreases as $N$ increases. Again, the change is abrupt.

The steps of figure 13 are due to the form of the curves of figure 12. Namely, in figure 12 we see that, for each value of $x$, only one of the three local minima at $N=14,30$ and 43 is the global minimum of the curve $D I$. For relatively small $x$, the global minimum $D I$ is the one at $N=43$, and this remains the global minimum as $x$ increases a little. But beyond a certain value of $x$, this minimum $D I$ becomes only a local minimum, while the global minimum is shifted to $N=30$. This value of $N$ continues to be a global minimum for increasing $x$ until the place of the global minimum changes again abruptly and it is taken by the minimum at $N=14$ (while the minima at $N=30,43$ are now only local minima).

It is to be noticed that this stepwise increase of the overall minimum $D I$ as a function of $N$ appears only in the resonance $\omega_{1} / \omega_{2}=4 / 3$ and not in the resonance $\omega_{1} / \omega_{2}=1 / 1$.

We have found a similar stepwise increase of $D I$ in the case $\omega_{1} / \omega_{2}=4 / 3$ and $H_{1}=x^{2} y^{2}$. On the other hand, a smooth variation of $D I$ with $N$ appears in all cases with $A=B=1$, even if the coupling term $H_{1}$ changes considerably. Such are the cases (4) (where $H_{1}=x^{2} y^{2}$ ), (5) (where $H_{1}=x^{2} y-y^{3} / 3$ ), and (6b) (where $H_{1}=-x y^{2}$ ).

Similar results appear in cases close to the resonances $\omega_{1} / \omega_{2}=4 / 3$ and $\omega_{1} / \omega_{2}=1 / 1$ and also close to higher order resonances.

\section{Integrable model (7)}

In this case there is a second exact integral (Bountis et al 1982) namely

$$
I=3\left(\dot{x}^{2}+x^{2}\right)-4 x^{2} y+4 \dot{x}(\dot{x} y-\dot{y} x)+x^{4}+4 x^{2} y^{2} .
$$




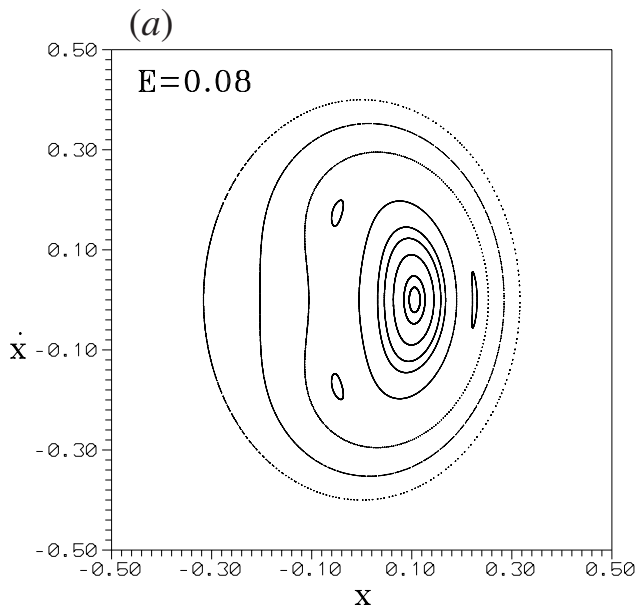

(b)

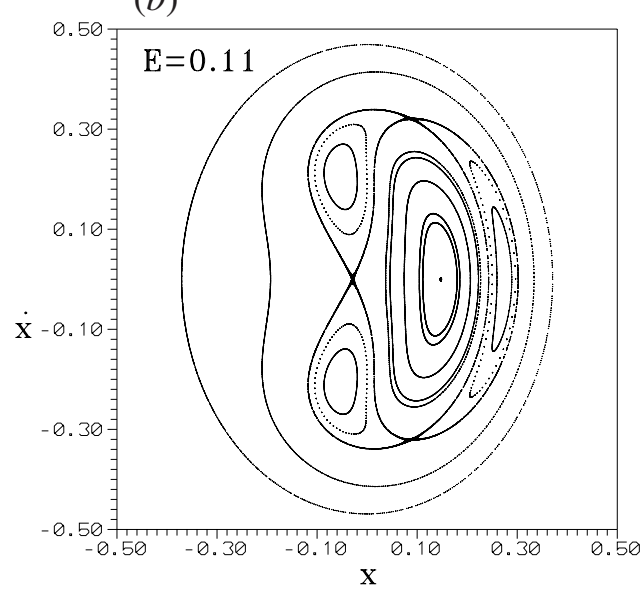

(c)

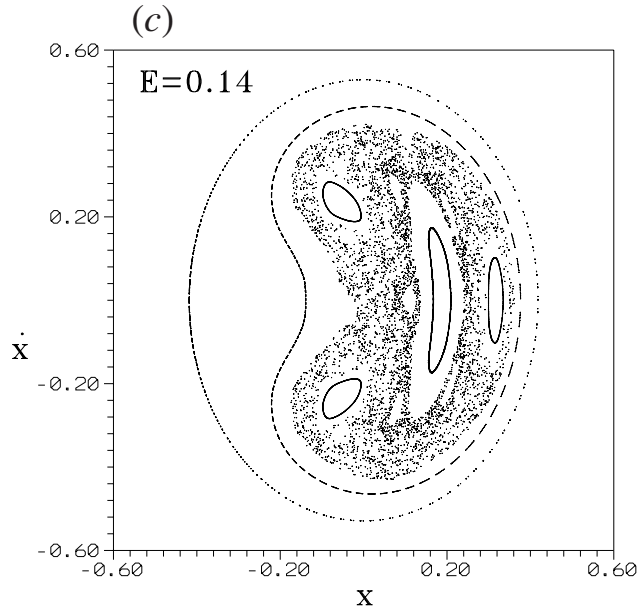

Figure 11. Poincaré surfaces of section (as in figure 1) for the Hamiltonian (6) with $A=1.6, B=$ 0.9 and $(a) E=0.08,(b) E=0.11$, and $(c) E=0.14$.

On a surface of section $x=0$, we have

$$
\dot{x}^{2}=2 E+4 y^{3}-y^{2}-\dot{y}^{2}
$$

and

$$
I=\dot{x}^{2}(3+4 y) \text {. }
$$

If we insert the value $\dot{x}$ given by equation (12) in equation (13), we find the equation of the invariant curves,

$$
\dot{y}^{2}=2 E+4 y^{3}-y^{2}-\frac{I}{(3+4 y)}
$$

for fixed $E$ and various values of $I$. In particular, one can find the periodic orbits and the boundary $\dot{x}^{2}=0$ on the Poincare surface of section.

A particular case, studied numerically by Kaluza and Robnik (1992), is with $E=0.004$. In this case the exact orbits give the invariant curves of figure 14(a). The theoretical invariant curves given by equation (14) agree completely with the empirical curves. 


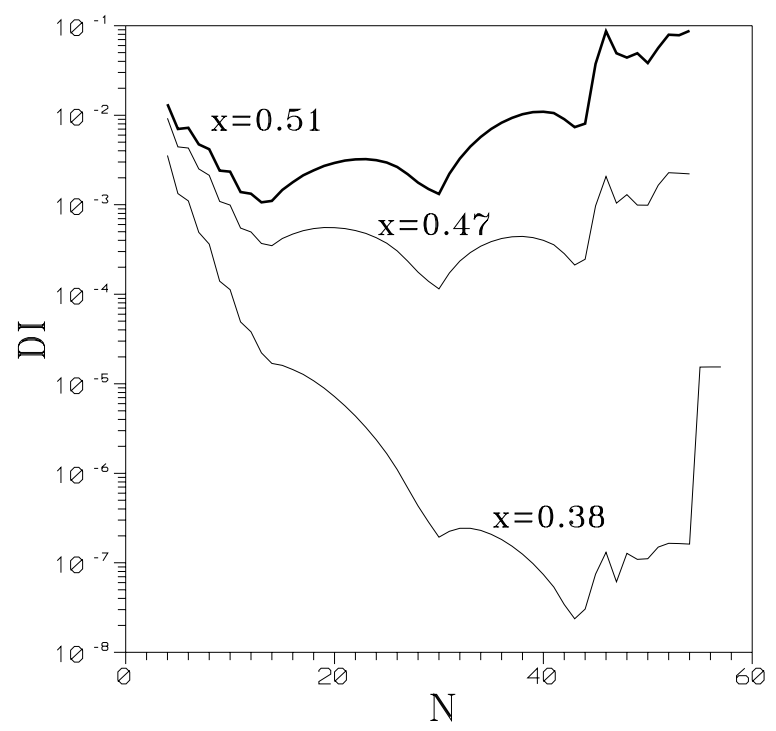

Figure 12. As in figure 4 for the Hamiltonian (6) with $A=1.6$ and $B=0.9$.

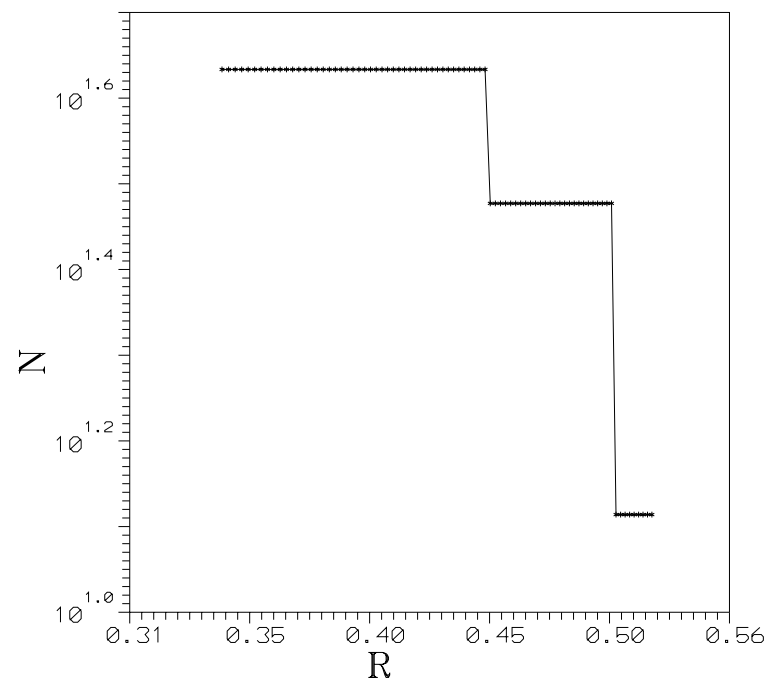

Figure 13. As in figure 5 for the Hamiltonian (6) with $A=1.6$ and $B=0.9$.

However, if we use the computer program of Giorgilli (1979), we find a disagreement between the invariant curves derived by the truncated formal second integral and the true invariant curves. The disagreement is more pronounced at high truncation orders (e.g. figure $14(b)$ for $N=50$ ), and it appears for $x \geqslant 0.083$.

This disagreement can be explained by remarking that Giorgilli's program gives the formal integral $I_{\mathrm{F}}$ only after the construction of Birkhoff's normal form. This construction gives the formal integral as a power series of the action variables of the non-linear system. For an integrable system, this series is convergent in some neighbourhood of the origin, but may 

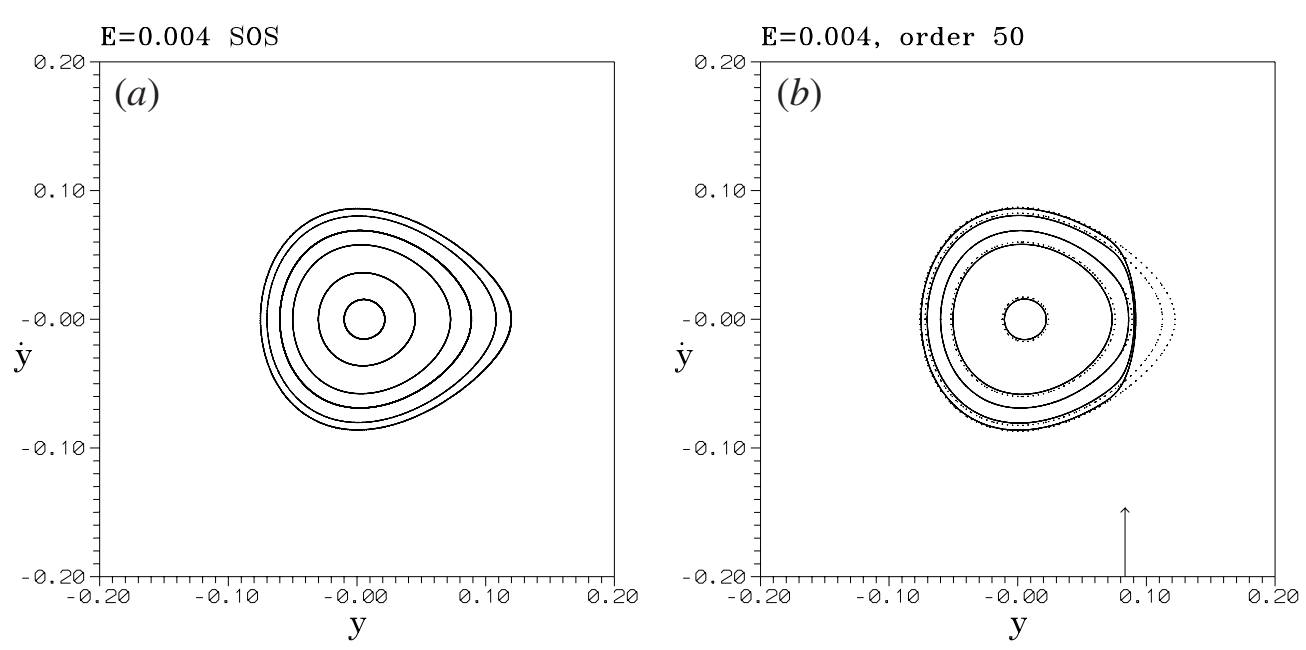

Figure 14. Poincaré $\operatorname{SOS}(y, \dot{y})$ (i.e. $x=0, \dot{x}=0$ ) for the Hamiltonian (7) and $E=0.004$. (a) Numerical orbits. (b) The exact invariant curves (level curves from the exact integral (equation (14), dashed lines) deviate from the level curves of the formal integral $I_{\mathrm{F}}$ (solid lines) for $y>0.083$.

not converge for large values of the variables. In general, it is only expected that the formal integral $I_{\mathrm{F}}$ is a function

$$
I_{\mathrm{F}}=I_{\mathrm{F}}(H, I)
$$

of the Hamiltonian $H$ and of the exact integral $I$. We have checked that, indeed, the formal integral found by the method of Giorgilli (1979) is of the form of equation (15) within the limit of the truncation order of our calculations.

In order to check the convergence of $I_{\mathrm{F}}$ we calculated, as in the previous sections, the logarithm of the variation of the truncated integral $\log (D I)$ as a function of the order $N$ for various values of $x$ (and $\dot{x}=0$ ).

Figure 15 gives the values of $\log (D I)$ for $x=0.02, x=0.04, x=0.07, x=0.08, x=$ 0.09 and $x=0.1$. We see that for small $x$, the values of $D I$ go to the limit of accuracy of our calculations $\left(10^{-11}\right)$ rather fast (for orders of truncation $N=18$ and $N=27$, respectively, for $x=0.02$ and $x=0.04$ ). For $x=0.07$ the convergence is slower and $D I$ has not yet reached the limiting value $10^{-11}$ at $N=50$. Nevertheless, the value of $D I$ is smaller than $10^{-8}$, i.e. the accuracy is sufficiently good.

On the other hand, for $x=0.09$ and $x=0.1$ the value of $D I$ increases with the order $N$. Therefore, no convergence is possible for $x$ equal to or larger than 0.09 . In the case $x=0.08$ the value of $D I$ changes little for large $N$. This is because this value of $x$ is close to the radius of convergence $x_{0}=0.083$ (see next section). In fact, for $x<x_{0}=0.083$ we have convergence, although slow, while for $x>0.083$ we have divergence of the formal integral.

In figure $14(b)$ the arrow marks the radius of convergence $y=0.083$. We see that some theoretical invariant curves cross this line. These curves are turned rather abruptly for larger $x$ and they are different from the real invariant curves of figure 14(a), represented by dashed lines in figure $14(b)$. Therefore, the theoretical curves derived from $I_{\mathrm{F}}$ (truncated at order 50) are not accurate, and in fact they do not agree with the exact theoretical invariant curves that are derived by means of the exact integral $I$. Nevertheless, for $0.033<x<0.09$ the deviations $D I$ of the integral $I_{\mathrm{F}}$ are smaller than $10^{-4}$ if the integral is truncated at order $N=50$ (although for $x=0.09$ they diverge for $N \rightarrow \infty$ ), thus the expected variations of 


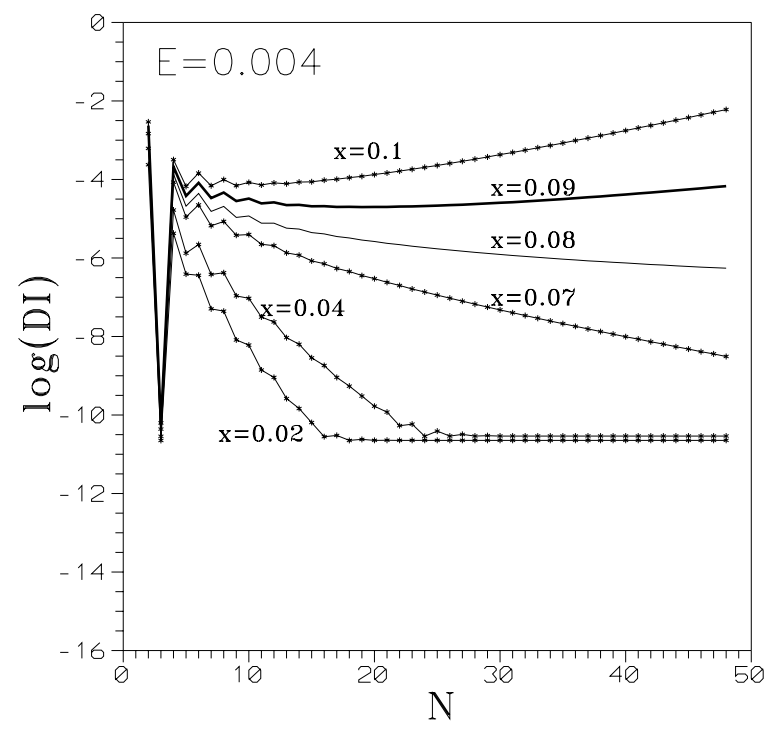

Figure 15. The variation $D I$ of the formal integral $I_{\mathrm{F}}$ as a function of the order $N$ in the case of the Hamiltonian (7) for $E=0.004$ and various values of $x$. The functions $I_{F}(N)$ tend to zero as $N \rightarrow \infty$ for $y \leqslant 0.08$, but they diverge for $y \geqslant 0.09$.

the theoretical invariant curves are not very large. However, orbits starting beyond $x=0.9$ give theoretical invariant curves that deviate appreciably from the exact invariant curves. These deviations demonstrate clearly the inappropriateness of the integral $I_{\mathrm{F}}$ for values beyond the radius of convergence.

\section{Radius of convergence}

One may try to evaluate a 'pseudo-radius of convergence' $R_{0}$ using the known methods of analysis. Having fixed a direction in the phase space, we evaluate two successive terms of the series $I$ at a fixed distance $R$ from the origin. According to the d'Alembert criterion, in the limit $N \rightarrow \infty$ we should have

$$
\frac{R I_{N-1}}{I_{N}} \rightarrow R_{0}
$$

Similarly, according to the Cauchy criterion we have

$$
\frac{R}{\sqrt[N]{I_{N}}} \rightarrow R_{0}^{\prime}
$$

If the series is convergent, then $R_{0}, R_{0}^{\prime}$ represent in both cases the radius of convergence. If the series is not convergent but has an asymptotic character, then the application of the above criteria to the first terms of the expansion may appear to converge to some limit, which we call 'pseudo-radius of convergence'.

In a simple example of a series representing a formal second integral of motion, Servizi et al (1983) found that the functions of this form decrease in steps as $N$ increases and tend to zero. This result shows that the series are not convergent for any value of $R$ different from zero. The step form of the function is due to the appearance of small divisors. In fact, the series giving the second integral has terms with divisors of the form $\left(m^{2} A-n^{2} B\right)$, and these divisors approach zero arbitrarily closely when $m$ and $n$ take appropriate values. In the 


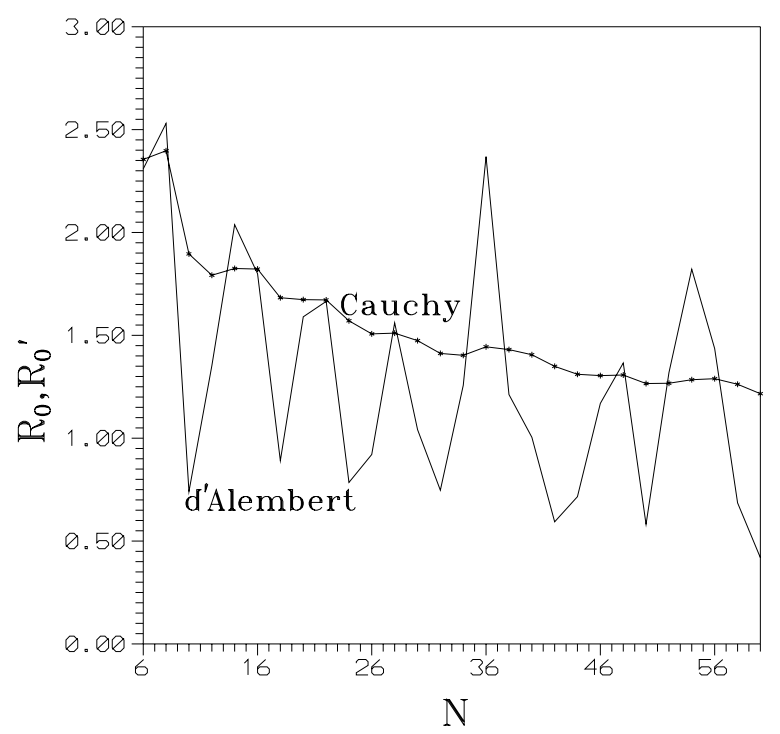

Figure 16. The pseudo-radius of convergence derived by the Cauchy and d'Alembert methods as a function of the order $N$ for the Hamiltonian (4).

case of an exact resonance (e.g. $A=B$ ), the construction of the formal integral avoids the obvious zero divisors, but terms with small divisors appear from time to time. When a term with a small divisor appears for the first time in the formal series, the values of the quantities $R_{0}=\left(R I_{N-1} / I_{N}\right)$ and $R_{0}^{\prime}=R \sqrt[N]{I_{N}}$ decrease abruptly and a step appears in each function $R_{0}$ or $R_{0}^{\prime}$.

In the following figures we calculate the radii $R_{0}$ (d'Alembert method) and $R_{0}^{\prime}$ (Cauchy method) as functions of $N$. In practice, for the d'Alembert criterion we considered the ratio of every two successive orders, namely

$$
R_{0}=\sqrt{\frac{R^{2} I_{N-2}}{I_{N}}}
$$

because in some models only the even order terms appear in the integral series. It should be noted that the value of $R_{0}$ or $R_{0}^{\prime}$ varies with the particular direction from the centre along which $R$ is calculated. In figure 16 we consider the particular direction $x=y$ and $\dot{x}=\dot{y}=0$.

Figure 16 gives the pseudo-radius of convergence for the Hamiltonian (4), as derived with the Cauchy and the d'Alembert methods, as a function of the order $N$ (equations (17) and (18), respectively).

We note that the Cauchy method gives a value of $R_{0}^{\prime}$ that varies rather smoothly with $N$, slowly decreasing and tending presumably to zero. On the other hand, the value of $R_{0}$ (d'Alembert) method has large oscillations, but decreases on average, also tending presumably to zero.

Both functions $R_{0}^{\prime}$ and $R_{0}$ do not show any plateaux as in the case of Servizi et al (1983). Similar results are found for the Hamiltonian (5) and for the Hamiltonian (6), both for $A=B=1$ and for $A=1.6, B=0.9$. In the last case the variations of $R_{0}$ (d'Alembert method) are very large.

The results are very different in the integrable case (Hamiltonian (7)) (figure 17). In this case both functions $R_{0}^{\prime}$ and $R_{0}$ tend smoothly to the same limit (the real radius of convergence) as $N \rightarrow \infty$. The convergence is much faster with the d'Alembert method. 


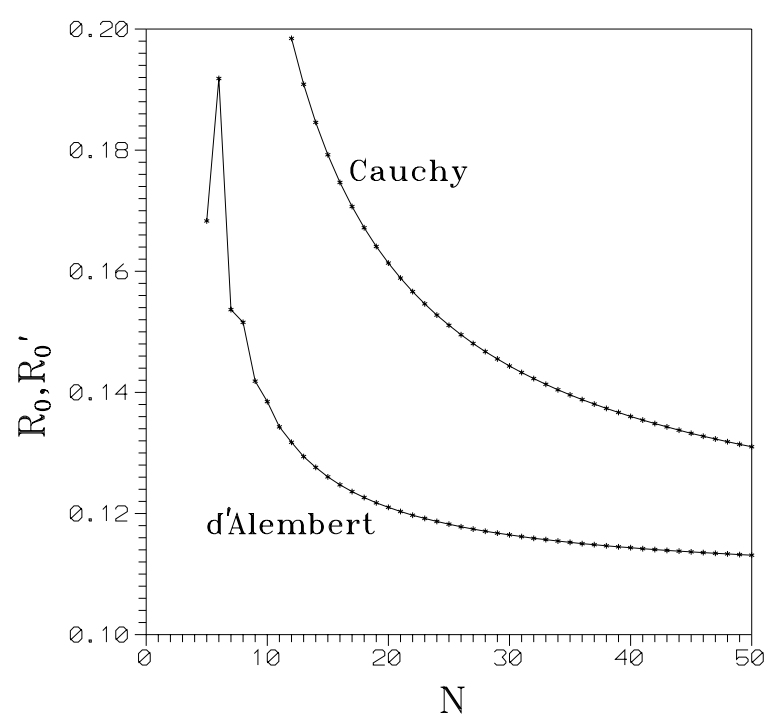

Figure 17. The pseudo-radius of convergence derived by the Cauchy and d'Alembert methods as a function of the order $N$ for the Hamiltonian (7). In this case both $R_{0}$ and $R_{0}^{\prime}$ tend to a finite radius of convergence.

An example shows why the d'Alembert method converges much faster than the Cauchy method. Consider the function

$$
I=\frac{a}{1-R}=a\left(1+R+R^{2}+\cdots\right) .
$$

The d'Alembert criterion gives

$$
\frac{I_{N-1}}{I_{N}}=\frac{1}{R}
$$

and we derive immediately (for any $N$, even for $N=1$ ) $R_{0}=1$.

On the other hand, the Cauchy criterion gives

$$
\frac{1}{\sqrt[N]{I_{N}}}=\frac{\sqrt[N]{a}}{R}
$$

This criterion gives $\lim _{N \rightarrow \infty} \sqrt[N]{a}=1$, but the convergence is slow, unless $a=1$. On the other hand, the d'Alembert criterion gives $R$ independently of the factor $a$.

In figure 17 we have taken $y=\dot{x}=R$ while $x=\dot{y}=0$. If we take other directions

$$
\dot{x}=c y=c R
$$

on the plane $(y, \dot{x})$, we find different values of $R_{0}$ for different directions $c$. Thus we calculate the domain of convergence in the plane $(y, \dot{x})$. This is the interior of the thick line in figure 18. The particular line $c=1$ is shown as a straight line in figure 18 and its intersection with the thick line gives a radius of convergence $R_{0}=y_{0}=\dot{x}_{0}=0.08$.

If the value of $c$ increases indefinitely, the line $\dot{x}=c y$ tends to the axis $y=0$. In this case the calculation of $R_{0}$ cannot be done directly from equation (16) because the odd terms with $y=0$ are zero. In this case only even order terms $I_{2 M}$ exist. Thus with an obvious generalization, we find

$$
\sqrt{\frac{I_{2 M-2}}{I_{2 M}}}=\frac{R_{0}}{R} .
$$

This formula is to be used also when $y$ is close to zero (i.e. $c$ is large). 


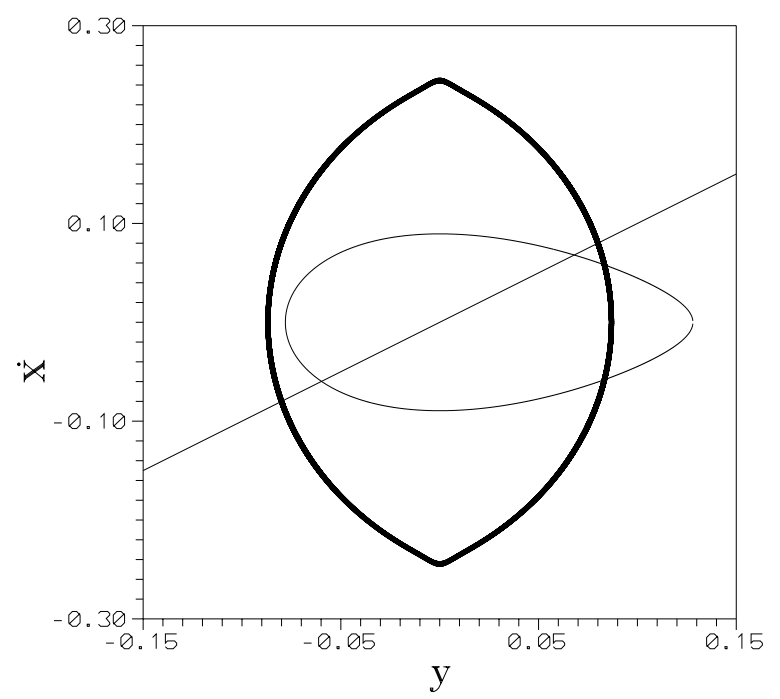

Figure 18. The region of convergence in the plane $(y, \dot{x})$ (thick line). The straight line is $\dot{x}=y$ and the elliptical-like line is given by equation (7) for $\dot{y}=0, x=0$ and $E=0.004$.

The value of $R_{0}$ is independent of the value of the energy $E$. However, it is possible to also define the radius of convergence $R=y$ as a function of $\dot{y}$ on a Poincaré surface of section $(y, \dot{y})$ for $x=0$ and fixed $E$. This is done by using equation (7) with constant energy. For example, if we set $\dot{y}=0$ (and also $x=0$ ) in equation (7) for $E=0.004$, we obtain a curve in the plane $(y, \dot{x})$. This is an elliptical-like curve in figure 18 . The maximum value of $R=y$ for convergence on the Poincaré surface of section $(y, \dot{y})$ with $\dot{y}=0$ is given by the intersection of this curve and the thick line of figure 18. This gives a radius of convergence $R_{0}=x_{0}=0.083$ which is indicated by an arrow in figure $14(b)$.

In a similar way we can find the radius of convergence $R=y$ for any value of $\dot{y}$ on the Poincaré surface of section $x=0, E=0.004$. This defines the domain of convergence of the formal integral on the Poincaré surface of section, which is limited by the thick line of figure 14(b). Only within this domain do the formal integral curves (solid curves in figure $14(b)$ ) coincide exactly with the real integral curves (dashed curves).

\section{Conclusions}

We consider formal integrals in simple 2D conservative dynamical systems. Our conclusions are the following.

(1) The formal integrals are not convergent in non-integrable cases. However, for relatively small energies, truncated forms of these integrals can be used in various applications as good approximations. In particular, one can find approximately the forms of the invariant curves on a Poincaré surface of section if there is not much chaos. We have checked that these statements are true up to the accuracy of our numerical calculations in three different systems consisting of two oscillators with a coupling term of the form $x^{2} y^{2}, x y^{2}$ and $x^{2} y-y^{3} / 3$ (Hénon-Heiles case).

(2) When the order of truncation increases beyond a certain order, the agreement is worse. The claim that in some cases one finds convergent behaviour of the formal integrals for 
small energies is not correct. The apparent convergence disappears for large orders of truncation.

(3) The variations of the formal integral $D I=I_{\max }-I_{\min }$ (truncated at a given order $N$ ) along an orbit depend on (a) the value of the energy, (b) the initial conditions and (c) the time interval of the calculation. The value of $D I$ has a minimum $D I$ at an optimal order of truncation $N$. This is independent of the time interval if this interval is sufficiently large.

(4) We studied the minimum value of $D I$ as a function of $\dot{x}$ for $x=0$ on the Poincaré section of the Hamiltonian (4), which has a particular symmetry around the centre, for various values of the energy. This function is approximately exponential near the centre. The exponent depends on the energy $E$. For relatively large $x$, the maximum $D I$ for any fixed $E$ occurs at a resonant periodic orbit $\dot{x}=\dot{x}_{B}$. But for small $x, D I$ has a local minimum for $\dot{x}=\dot{x}_{B}$.

(5) The order $N$ of the best truncation is a decreasing function of the distance $R$ from the centre. This is consistent with the Nekhoroshev theorem about the best truncation of formal integrals. In some models (e.g. $A=B$ and various forms of the term $H_{1}$ ) the decreasing function $N(R)$ is smooth, with an average power law, while in other cases (e.g. $A=1.6, B=0.9$ ) the decrease is in abrupt steps. This phenomenon may be related to the particular resonant structure of each model.

(6) In an integrable case the computer algorithm does not always give the simplest form $I$ of the second integral; instead, it gives a function $I_{\mathrm{F}}$ of $I$ and of the energy $E$ which may or may not converge. The simplest form $I$ gives exactly the empirical invariant curves. However, the function $I_{\mathrm{F}}$ gives correct results only up to the radius of convergence.

(7) A 'pseudo-radius of convergence' can be calculated by the d'Alembert method ( $R_{0}$, found by taking the ratio of successive terms of the formal integral), or by the Cauchy method ( $R_{0}^{\prime}$, found by taking the $N$ th root of the $N$ th term of the formal integral). The pseudo-radius of convergence is a function of the order $N$. Its limit when $N \rightarrow \infty$ is finite in integrable cases and zero in non-integrable cases. In non-integrable cases the variation of $R_{0}^{\prime}$ (Cauchy method) is smooth, while the variation of $R_{0}$ (d'Alembert method) is large. Both $R_{0}^{\prime}$ and $R_{0}$ tend slowly to zero.

(8) In an integrable case the Cauchy and d'Alembert criteria give a finite radius of convergence. The convergence with the d'Alembert criterion is much faster than that with the Cauchy criterion. We found a method to calculate the radius of convergence for various energies and various initial conditions.

\section{Acknowledgments}

This research was supported in part by the Research Committee of the Academy of Athens (grant 200/493). CE was supported in part by the Greek State Scholarship Foundation.

\section{References}

Birkhoff G D 1927 Dynamical Systems (Providence, RI: American Mathematical Society)

Bountis T, Segur H and Vivaldi F 1982 Phys. Rev. A 251257

Celletti A and Giorgilli A 1991 Celest. Mech. Dyn. Astron. 5031

Cherry T M 1924a Proc. Camb. Phil. Soc. 22325

Cherry T M 1924b Proc. Camb. Phil. Soc. 22510

Contopoulos G 1960 Z. Astrophys. 49273

Contopoulos G 1963 Astron. J. 68763

Contopoulos G 1970 Astron. J. 7596

Contopoulos G 1971 Astron. J. 76147 
Contopoulos G, Efthymiopoulos C and Voglis N 2000 Celest. Mech. Dyn. Astron. 78243

Contopoulos G, Giorgilli A and Galgani L 1978 Phys. Rev. A 181183

Contopoulos G and Moutsoulas M 1965 Astron. J. 70817

Contopoulos G, Papadaki H and Polymilis C 1994 Celest. Mech. Dyn. Astron. 60249

Giorgilli A 1979 Comput. Phys. Commun. 16331

Giorgilli A 1988 Ann. Inst. H Poincaré 48423

Giorgilli A, Delshams A, Fontich E, Galgani L and Simò C 1989 J. Differ. Eqns. 77167

Giorgilli A and Galgani L 1978 Celest. Mech. 17267

Giorgilli A and Skokos Ch 1997 Astron. Astrophys. 317254

Gustavson F 1966 Astron. J. 71670

Hénon M and Heiles C 1964 Astron. J. 6973

Kaluza M and Robnik M 1992 J. Phys. A: Math. Gen. 255311

Littlewood J E 1959a Proc. Lond. Math. Soc. 9343

Littlewood J E 1959b Proc. Lond. Math. Soc. 9525

Nekhoroshev N N 1977 Russ. Math. Surv. 321

Roels J and Hénon M 1967 Bull. Astron. Soc. 2267

Servizi G, Turchetti G, Benettin G and Giorgilli A 1983 Phys. Lett. A 9511

Siegel C L 1941 Ann. Math. 42806

Skokos Ch and Dokoumetzidis A 2001 Astron. Astrophys. 367729

Whittaker E T 1916 Proc. R. Soc. Edin. 3795

Whittaker E T 1937 A Treatise on the Analytical Dynamics of Particles and Rigid Bodies (Cambridge, MA: Cambridge University Press) 